\title{
A NEW MODEL FOR HIGHWAY CONSTRUCTION SITE LAYOUT PROBLEM USING A HYBRID OPTIMIZATION APPROACH
}

\author{
Ibrahim H. Hashim ${ }^{1}$ and A. A. Mousa ${ }^{2}$ \\ ${ }^{\prime}$ Civil Engineering Department, Faculty of Engineering, Menoufia University \\ ${ }^{2}$ Basic Engineering Sciences Department, Faculty of Engineering, Menoufia University
}

\begin{abstract}
:
Undoubtedly, site layout is a very important planning problem for the construction of any project. A new approach to solve the multi-objective construction site layout (MCSL) problem is presented. It is based on the combination of Genetic Algorithm (GA) and the TOPSIS technique. MCSL is considered with assigning of predetermined facilities into a number of predetermined places. The proposed approach has two main objectives. The first is to minimize overall distance, while the second is to reduce the number of crossing paths between different facilities. Also, the approach has two characteristic features. Firstly, a new chromosome's structure is introduced, which is adopted as it is capable to representing all possible feasible solutions. Secondly, the algorithm is an iterative multi-objective genetic algorithm with an external population of Pareto optimal solutions that best conform a Pareto front. To help the decision makers to extract the best compromise solution from a finite set of alternatives, a TOPSIS (Technique for Order Performance by Similarity to Ideal Solution) method is adopted. A case study for a simple highway project is presented to illustrate the application of the proposed model.

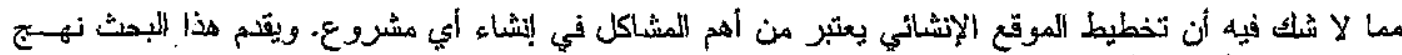

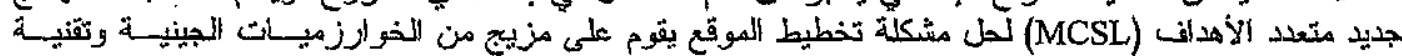
TOPSIS

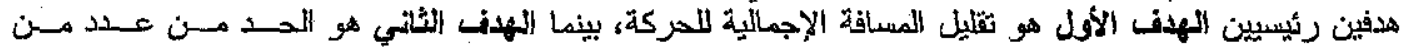

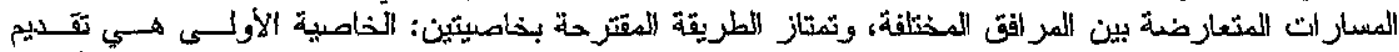

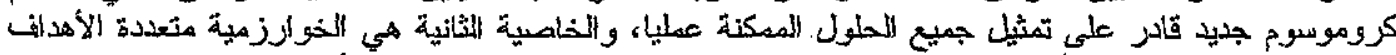

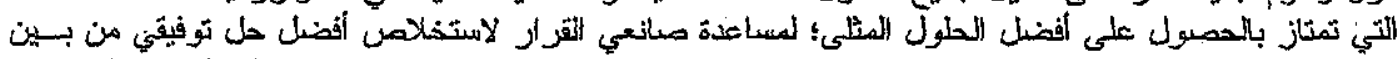

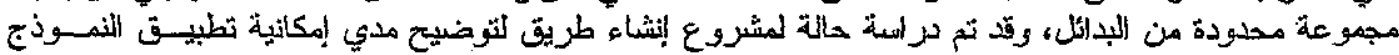
المقترح ميداثياً.
\end{abstract}

Keywords: Construction site layout problem; Highway construction; Multi-objective Genetic Algorithms; TOPSIS.

\section{INTRODUCTION}

Construction site layout is a very important planning problem. The main aim of site layout is to place temporary facilities both geographically and at the correct time such that the construction work can be performed efficiently and safely as well as with minimal costs. During the last decade, evolutionary approaches (EAs) such as genetic algorithms have been used extensively for the construction site layout problem [1-5]. Genetic Algorithms have proved themselves capable of resolving combinatory optimization problems that would be extremely complicated for conventional techniques. This paper proposes a hybrid multi-objective genetic optimization model for highway construction site layout. The proposed approach has two characteristic features. Firstly, a new chromosome's structure is introduced, which is adopted as it is capable to representing all possible feasible solutions. A criterion was designed in order to keep the feasibility of the chromosome. Based on this criterion the crossover and mutation were modified and implemented to generate feasible chromosomes. Secondly, the algorithm is an iterative multiobjective genetic algorithm with an external population of Pareto optimal solutions that best match a Pareto front.

This paper consists of five sections. In the next section, a formulation of the site layout problem is proposed in terms of a sequencing problem that is suitable for solution using the proposed algorithn. Section three provides a theoretical background and fundamentals of multi-objective optimization, iterative multi-objective search algorithm, Genetic algorithm and TOPSIS method. Section four, describes a practical application to a simple case study for a highway construction project. Solutions 
are expounded in the fifth section, and conclusions are drawn in the sixth section.

\section{THE CONSTRUCTION SITE LAYOUT PROBLEM}

There is no doubt that the demand for road transport has been increasing rapidly due to the increase of motorization, urbanization and population growth. To. meet this significant increase, a large number of highway construction projects are either on-going or being planned. Planning an effective site layout of such highway projects is a significant, demanding and challenging task that should be carefully analyzed and executed by construction planners and decision-makers. For these reasons, the proposed method was applied to a highway construction project, as a case study.

The objective of construction site layout is to allocate appropriate locations and areas for temporary sitelevel facilities such as warehouses, job offices, workshops and batch plants. The main challenges in managing construction sites, especially in the case of large projects, are to reduce the overall movement distances of construction equipments/materials between their temporarily places as well as to prevent/reduce the crossing paths of these movements. Thus, the construction work can be performed satisfactorily, safely and economically. Tommelein [6] defined the benefits from laying a site well as " $A$ well-organized site facilitates inventory control, cuts travel times, reduces' noise and dust, prevents obstructions and interferences, increase safety and security, and improves site access".

Significant research has been made for optimizing site layout planning, leading to a number of models that employed a variety of approaches such as artificial intelligence [7], annealed neural networks [8], dynamic layout planning [9], geographic information systems [10], genetic algorithms [11], ant colony [12], and approximate dynamic programming [13].

Considering only Genetic Algorithm (GA) Models, the first model developed applying GA to facility layout problem was probably EvoSite: Evolutionbased model for site layout planning by Hegazy and Elbeltagi [11]. This model considers the total travel distance as the only objective function. Using GA, Zouein et al. [14] developed a model to solve site layout problems with unequal-sized and constrained facilities. The objective function used in this model was mainly to minimize the total transportation cost [14]. Mawdesley and Al-Jibouri [3] presented another model applying GA to construction site layout problems. This model was more comprehensive and generic compared to the previous ones even though it is noted that the limitations of the facilities and site areas to be rectangle in shape and their sides to parallel with each other. Many other efforts to applying GA in such problems may include Tang and Tom [1], Osman et al. [2], and Cheung et al. [4].

Although most of theses models have contributed to site layout representations, they are still simplifying such problem. All of these models were concentrated on reducing the travel costs/distances and therefore they all are incapable of maximizing construction safety by minimizing/preventing conflicting paths between equipments. The proposed approach presents optimization system which has two main objectives; to minimize the conflicting paths in addition to minimize overall distances which ensure more safe and low time. Optimization of the aboveformulated objective functions using multi-objective evolutionary algorithms yields not a single optimal solution, but a set of Pareto optimal solutions, in which one objective cannot be improved without sacrificing other objectives. For practical applications, however, it is necessary to select one solution, which will satisfy the different goals to some extent. For this reason, TOPSIS method [15] is implemented to help decision maker to choose the best solution which called best compromise solution.

\section{THEORETICAL BACKGROUND}

The main objectives of this work is to develop a new optimization system to solve the multi-objective construction site layout (MCSL), based on the combination of Genetic Algorithm (GA) and the TOPSIS technique. However, before explaining such approach, this section provides a theoretical background of the techniques and procedures used. It discusses the fundamentals of the multi-objective optimization, the structure of an iterative multiobjective search algorithm, the Genetic algorithm and the TOPSIS method.

\subsection{Multi-objective Optimization}

A general multi-objective optimization problem (MOP) [16] is expressed, as follows:

$$
\begin{aligned}
\operatorname{Min} F(x)= & \left(f_{1}(x), f_{2}(x), \ldots, f_{k}(x)\right)^{T} \\
& s t . \quad x \in S \\
x= & \left(x_{1}, x_{2}, \ldots, x_{n}\right)^{T}
\end{aligned}
$$

where:

$$
\begin{aligned}
& \left(f_{1}(x), \ldots, f_{k}(x)\right) \text { the } k \text { objectives functions; } \\
& \left(x_{1}, x_{2}, \ldots, x_{n}\right) \text { the } n \text { optimization parameters; and } \\
& S \in R^{n} \text { the solution or parameter space. }
\end{aligned}
$$

Because $F(x)$ is a vector, there is no unique solution to this problem; instead, the concept of noninferiority (also called Pareto optimality) must be used to characterize the objectives. A non-inferior solution is one in which an improvement in one objective requires a degradation of another. This 
concept will be defined more precisely in the following subsection.

(Pareto optimal solution [16]): $x^{*}$ is said to be a Pareto optimal solution of MOP if there exists no other feasible $x \quad$ (i.e., $x \in S$ ) such that, $f_{j}(x) \leq f_{j}\left(x^{*}\right)$ for all $j=1,2, \ldots, k$ and $f_{j}(x)<f_{j}\left(x^{*}\right)$. for at least one objective function $f_{j}$.

The final goal in a MOP is to find as many as possible such Pareto optimal solutions to represent trade-off information among different objectives.

\subsection{Structure of an Iterative Multi-objective Search Algorithm}

An abstract description of a generic iterative search algorithm is given in algorithm 1 [i7]. The integer $t$ denotes the iteration count, the n-dimensional vector $f^{(t)} \in F$ is the sample generated at iteration $t$ and the set $A^{(t)}$ will be called the archive at iteration $t$ and should contain a representative subset of the samples in the objective space $\mathrm{F}$ generated so far. To simplify the notation, the samples are represented by $n$-dimensional real vectors $f$ where each coordinate represents one of the objective values, as in Fig. 1.

Algorithm I: Iterative search procedure

1.t@0

2. $A^{(0)}=0$

3. while terminate $\left(\mathrm{A}^{(t)}, t\right)=$ false do

4. $t @+1$

5. $f^{(1)} @ g$ generate 0

6. $A^{(1)} @$ update $\left(A^{(1-1)} f^{(1)}\right) \quad$ \{update archive\}

7. end while

8. Ontput : $A^{(1)}$

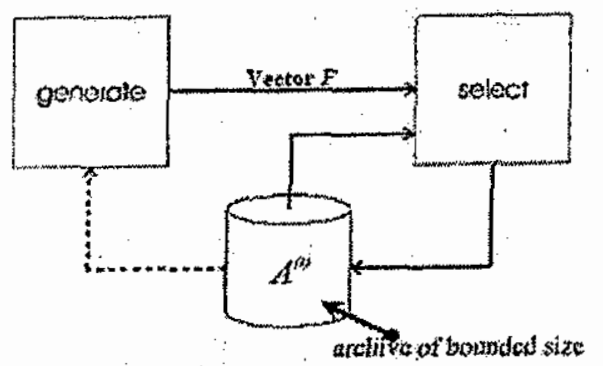

Fig. 1. Block diagram of archive/selection strategy

The purpose of the function generate is to generate a new solutions in each iteration $t$, possibly using the contents of the old archive set $A^{(t-1)}$. The function update gets the new solutions $f^{(t)}$ and the old archive set $A^{(t-1)}$ and determines the updated one, namely $A^{(t)}$. In general, the purpose of this sample storage is to gather 'useful' information about the underlying search problem during the run. Its use is usually two-fold; on the one hand it is used to store the 'best' solutions found so far, on the other hand the search operator exploits this information to steer the search to promising regions.

\subsection{Genetic algorithms}

Genetic Algorithms (GAs) came to the fore in the late 60 's through the work of Holland [18], the first person to put this computational development on a firm theoretical footing [19]. GAs are a division of the rapidly growing areas of artificial intelligence [19-21]. They are inspired by Darwin's theory of biological evolution. By mimicking this process, genetic algorithm are able to "evolve" solutions to real world problems. In this approach, the variables are represented as genes on a chromosome. GAs feature a group of candidate solutions (which is called population) on the response surface. Through natural selection and the genetic operators, mutation and recombination, chromosomes with better fitness are found. Natural selection guarantees that chromosomes with better fitness will propagate in the future populations. Using the recombination operator the GA combines genes from two parent chromosomes to form children (new chromosomes) that have a high probability of having better fitness than their parents. Mutation allows new areas of the response surface to be explored. GAs offer a generation improvement in the fitness of the chromosomes and after many generations will create chromosomes containing the optimized variable settings. One of the hallmarks of GAs is that they work with a population of possible solutions, in contrast to other heuristic search methods, which work with a single solution. Figure 2 shows a flowchart of the working of a GA.

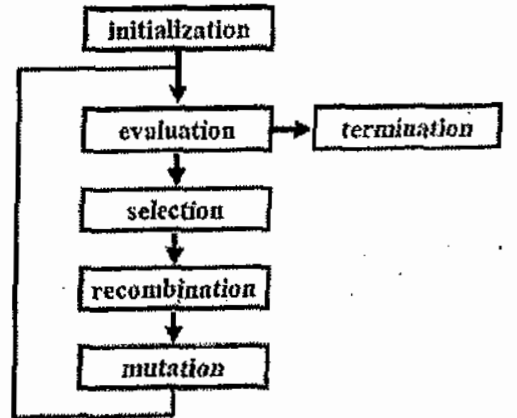

Fig. 2. Main flowchart of SGA.

\subsection{TOPSIS Method}

TOPSIS method, given by Hwang and Yoon [15], has the ability to identify the best alternative from a finite set of alternatives quickly. It stands for 
"Technique for Order Preference by Similarity to the. Ideal Solution". It is based upon the concept that the chosen alternative should have the shortest distance from the positive ideal solution and the farthest from the negative ideal solution. TOPSIS can incorporate relative weights of criterion importance. The idea of TOPSIS can be expressed in a series of steps [22], as follows:

1) Obtain performance data for $n$ alternatives over $M$ criteria $x_{i j}(i=1, \ldots \ldots, n, j=1, \ldots ., K)$;

2) Calculate normalized rating (vector normalization is used) $r_{i j}$;

3) Develop a set of importance weights $W_{k}$, for each of the criteria. The basis for these weights can be anything, but, usually, is ad hoc reflective of relative importance $V_{i j}=w_{j} \cdot r_{i j}$;

4) Identify the ideal alternative (extreme performance on each criterion, $S^{+}$), $S^{+}=\left\{v_{1}^{+}, v_{2}^{+}, \ldots, v_{j}^{+}, . ., v_{k}^{+}\right\}$

$$
=\left\{\left(\max v_{\mathrm{ij}} \mid j \in J_{1}\right),\left(\min \mathrm{v}_{\mathrm{ij}} \mid j \in J_{2}\right), i=1, \ldots, n\right\}
$$

5) where: $J_{1}$ is a set of benefit attributes; and $J_{2}$ is a set of cost attributes;

6) Identify the nadir alternative (reverse extreme performance on each criterion, $S^{-}$)

$$
\begin{aligned}
S^{-} & =\left\{v_{1}^{-}, v_{2}^{-}, . ., v_{j}^{-}, . ., \nu_{k}^{-}\right\} \\
& =\left\{\left(\operatorname{minx} v_{\mathrm{ij}} \mid j \in J_{1}\right),\left(\max \mathrm{v}_{\mathrm{ij}} \mid j \in J_{2}\right), i=1, \ldots, n\right\} ;
\end{aligned}
$$

7) Develop a distance measure over each criterion to both ideal $\left(D^{+}\right)$and nadir $\left(D^{-}\right)$,

$$
D_{i}^{+}=\sqrt{\sum_{j}\left(v_{i j}-v_{j}^{+}\right)^{2}}, \quad D_{i}^{-}=\sqrt{\sum_{j}\left(v_{i j}-v_{j}^{-}\right)^{2}}
$$

8) For each alternative, determine a ratio $R$ equal to the distance to the nadir divided by the sum of the distance to the nadir and the distance to the ideal,

$$
R=\frac{D^{-}}{D^{-}+D^{+}}
$$

9) Rank alternative according to $R$ ratio (in Step 7) in descending order, and

10) Recommend the alternative with the maximum ratio.

\section{THE PROPOSED ALGORTTHM}

In the following sub-sections, the proposed hybrid approach for solving the MSCL is presented.

\subsection{Initialization Stage}

The genetic representation is a kind of data structure which represents the candidate solution of the problem in coding space. In order to form the appropriate design of chromosome using the proposed approach, first consider each chromosome consists of a sequence of $n$ positions ( $n$ is the number of construction facilities), as in Fig. 3.

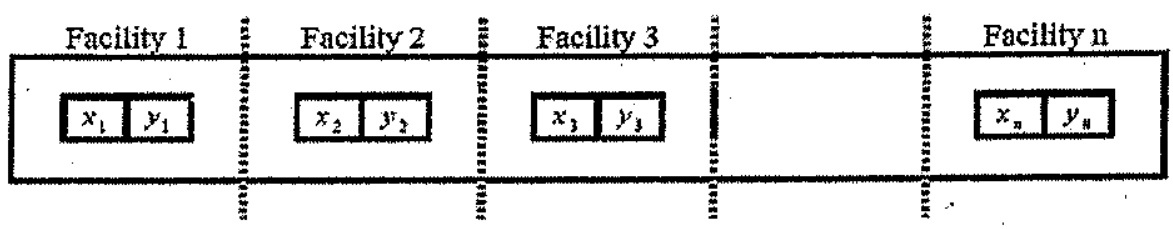

Fig. 3. Structure of chromosome for MSCL with $n$ facilities

As an example, a construction site layout problem in a highway project is proposed, as shown in Fig. 4. Tolerance space is defined to indicate that each construction facility center must . away from the highway by its area radius (see Fig. 5). Consequently, the feasible region refers to the areas that are available to accommodate all construction facilities, as in Fig. 4.

\subsection{Evaluation of Non-Dominated Solutions}

A population of size $N$ can be evaluated according to non-domination concept. Consider a set of population members, having $K(K>1)$ objective function values. The following procedure explains the algorithm by which the non-dominated set of solutions can be found.

Step 0: Begin with $\mathrm{i}=\mathrm{i}$.
Step 1: For all $j=1,2, . ., N$ and $j \neq i$, compare solutions $x^{i}$ and $x^{j}$ for domination.

Step 2: If for any $j . x^{i}$, is dominated by $x^{j}$, mark $x^{i}$ as "dominated".

Step 3: If all solutions (that is, when $i=N$ is reached) in the set are considered, Go to Step 4, else increment $i$ by one and Go to Step 1 .

Step 4: All solutions that are not marked "dominated" are non-dominated solutions.

The algorithm initially locates an externally finite size archive of observed non-dominated solutions. 


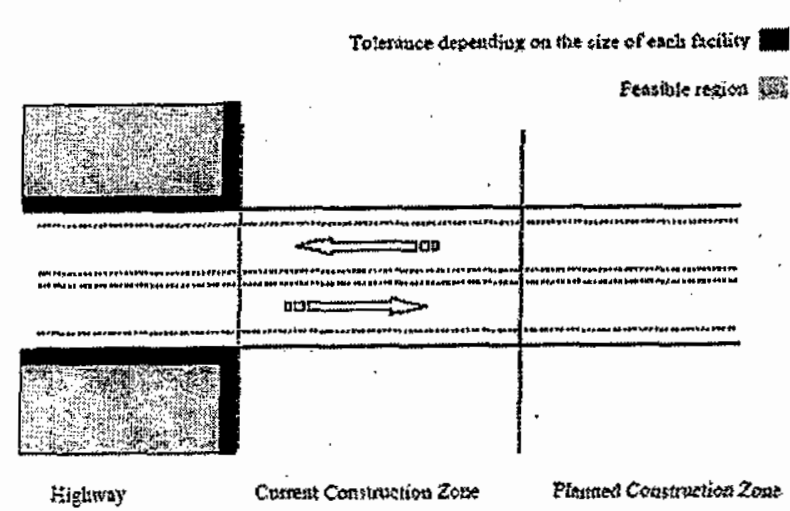

Fig. 4. Example of a site layout for a highway project

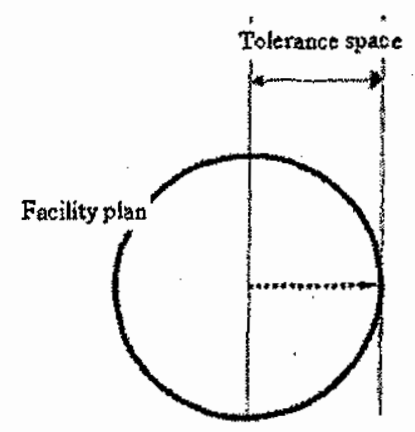

Fig. 5. Tolerance space

\subsection{Selection Stage}

Selection (reproduction) operator is intended to improve the average quality of the population by giving the high-quality chromosomes a better chance to get copied into the next generation. The principle behind GAs is essentially Darwinian natural selection. The selection directs GA search towards promising regions in the search space. A randomweight approach $[5,18]$ is proposed to obtain a variable search direction towards the Pareto frontier. Suppose that it is going to maximize $\mathrm{k}$ objective function, the weighted-sum objective is given as follows:

$f(x)=w_{1} f_{1}(x)+\ldots .+w_{k} f_{k}(x)=\sum_{i=1}^{k} w_{i} f_{i}(x)$

where:

$x$ is a string (i.e., individual );

$f(x)$ is a combined fitness function;

$f_{i}(x)$ is the $i$-th objective function; and

$\left\{w_{i} \mid \sum_{i=1}^{k} w_{i}=1\right\}$ is a constant weight for $f_{i}(x)$.

A roulette wheel selection is employed as a selection mechanism in this study. In such selection mechanism, the individuals on each generation are selected for survival into the next generation according to a probability value proportional to the ratio of individual fitness over total population fitness. This means that on average the next generation will receive copies of an individual in proportion to the importance of its fitness value. The probability of variable selection is proportional to its fitness value in the population, according to the following formula:

$$
p(x)=\frac{f(x)-f_{\text {Min }}(\psi)}{\sum_{x \in \psi}\left\{f(x)-f_{M i n}(\psi)\right\}}
$$

where:

$p(x)$ is the selection probability of a string $x$ in a population $\psi$; and

$f_{\text {Min }}(\psi)=\operatorname{Min}\{f(x) \mid x \in \psi\}$

\subsection{Crossover Operators}

The goal of crossover is to exchange information between two parent chromosomes in order to produce two new offspring for the next population. A modified uniform crossover is presented, where it is possible to choose two parent from the mating pool that have the same number of facilities in the right to (or in the left to) the highway. In the example problem in Fig. 6 there are six facilities; three facilities from the first parent in the right to the highway exactly as from the second parent. Therefore these two parents are suitable for crossover operator. It is interesting here to note that all offspring's chromosome are feasible.
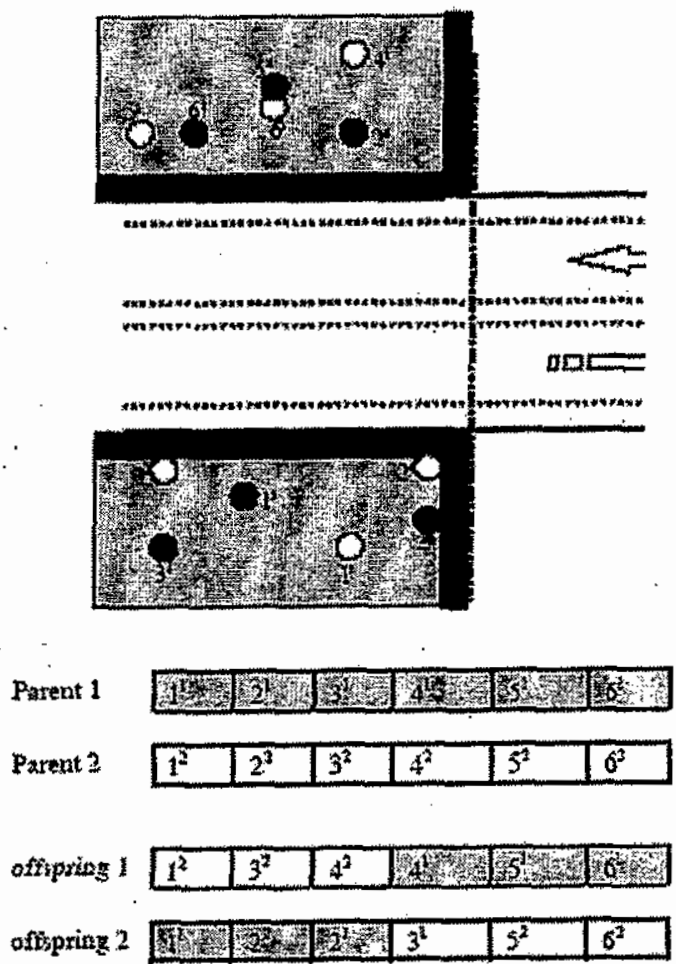

Fig. 6. Graphs visualizing the crossover operators. 


\subsection{Mutation Operators}

A mutation operator is a random process where one genotype is replaced by another to generate a new chromosome. Such a mutation operator first select a gene randomly from $i$-th gene and then replace it with a random new position from the feasible region except the location of the other facilities location, as shown in Fig. 7.

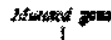

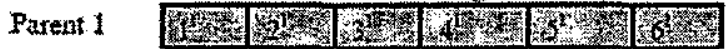

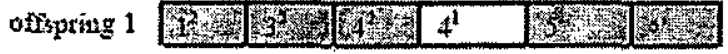

Fig. 7. Graphs visualizing the mutation operators.

In Fig. 7, there are 6 facilities; the fourth one is mutated to get new facility position. Through this mutation operator, the population's feasibility was preserved.

\subsection{Update Function for Pareto Set.}

In order to ensure convergence to the true Paretooptimal solutions, the study concentrated on how elitism could be introduced in the algorithm. So, an archiving/selection strategy that guarantees at the same time progress towards the Pareto-optimal set and covering the whole range of the non-dominated solutions is proposed. This can be done using update function where, it gets the new population $P^{(t)}$ and the old archive set. $A^{(t-1)}$ and determines the updated one, namely $A^{(t)}$.This is explained in algorithm 1 , where the generated point $x$ has three possibilities: to be rejected, to be replaced by any point in the archive, or to be added in the archive.

\subsection{TOPSIS Implementation}

Optimization of the above-formulated objective functions using multi-objective genetic algorithms yields not a single optimal solution, but a set of Pareto optimal solutions. TOPSIS technique is used to select one solution, which will satisfy the different goals to some extent, where, the only subjective input needed is weights which reflect the degree of satisfactory of each objective.

1) Obtain the set of $n$ Pareto optimal solutions.

2) Calculate normalized rating (vector normalization is used).

3) Develop a set of importance weights $W_{k}$, for each of the criteria. The basis for choosing these weights can be anything, but, usually, is ad hoc reflective of relative importance of each criterion.

4) Identify the ideal and nadir alternative, as explained in Section 3.4.

5) Develop a distance measure over each criterion,
6) For each alternative, determine a ratio $R$.

7) Rank alternative according to $R$ ratio in descending order.

\section{SIMULATION RESULTS AND DISCUSSIONS}

In this Construction site layout problem, Multiobjective optimization problem is introduced, the first objective is to minimize the total cost of movements of construction equipment from facilities $i$ to $j$. This cost of movernents will be represented as the product of the traveling distances between the facilities and the frequency of traveling.

$$
\operatorname{Min} Z=\sum_{i, j=1}^{n} f^{*} d_{i j}, \quad f=\left[\begin{array}{cccccc}
0 & 2 & 2 & 1 & 4 & 9 \\
2 & 0 & 7 & 4 & 9 & 6 \\
2 & 7 & 0 & 7 & 8 & 5 \\
1 & 4 & 7 & 0 & 5 & 7 \\
4 & 9 & 8 & 5 & 0 & 3 \\
9 & 6 & 5 & 7 & 3 & 0
\end{array}\right]
$$

where:

$f$ is the frequency of movements (the same as those assumed by AbdelRazig et al. [12]); and

$d$ is the distance between facilities.

The second objective is to minimize the number of intersecting paths (i.e. conflicting paths) between different facilities. This construction layout problem indicates that 6 facilities are must located in 6 different locations. The Facilities are: (1) site office, (2) labor trailer (3) loader equipments, (4) storage, (5) trucks storage area, and (6) backhoes storage area. It is assumed that each facility has an area of a radius of $7.5 \mathrm{~m}$.

The problem formulation:

$$
\operatorname{Min} Z_{1}=\sum_{i, j=1}^{n} f * d(i, j)
$$

$\operatorname{Min} Z_{2}=$ number of Conflicting Pathes

st.

$$
\begin{aligned}
& d(i, j) \geq r_{i}+r_{j} \\
& 0 \leq x_{i} \leq X_{\max }, \\
& 25+r_{i} \leq\left|y_{i}\right| \leq 75-r_{i} .
\end{aligned}
$$

where:

$$
r_{i}: i \text {-th facility radius; }
$$

$\left(x_{i}, y_{i}\right): i$-th facility center;

$d(i, j):$ is the distance between facility $i$ to facility $j$; and

$X_{n a x}$ : the available range where the facilities can be located

Note that the zero of $y$-axis is in the middle of the roadway 
The proposed algorithm was implemented on 2.7MHz PC using MATLAB 6.5. Table 1 lists the parameter setting used in the algorithm.

Table 1. Parameter values used for all runs.

\begin{tabular}{lc}
\hline Parameter & Value \\
\hline Problem size & 100 \\
Number of objective & 2 \\
Mutation rate & 0.50 \\
Crossover rate & 0.98 \\
Maximum generation & 50 \\
\hline
\end{tabular}

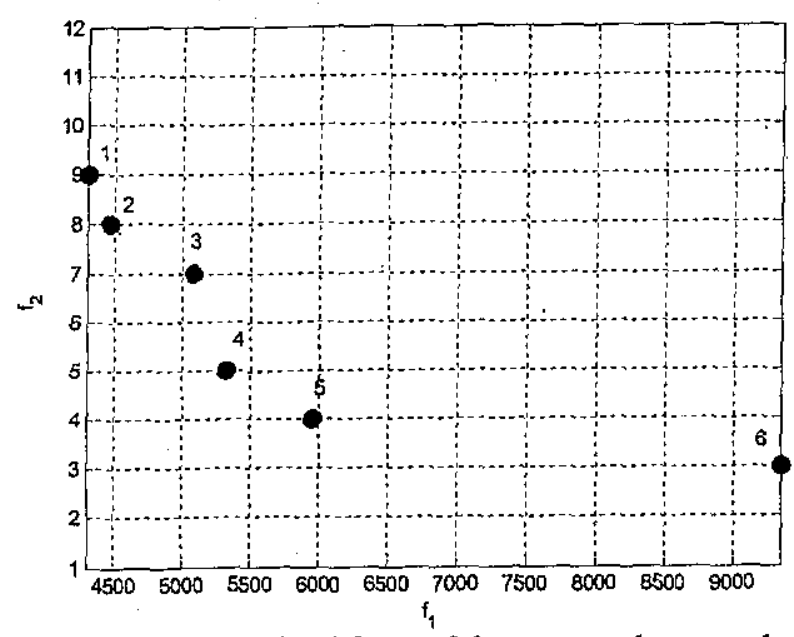

Fig. 8. Pareto-optimal front of the proposed approach

As shown in Fig. 8, the algorithm worked well in both distribution and spread. Also, the methods keep track of all the feasible solutions found by iteratively update the archive content during the optimization. Figure 9 outlines the locations of the different facilities in nine different solutions.
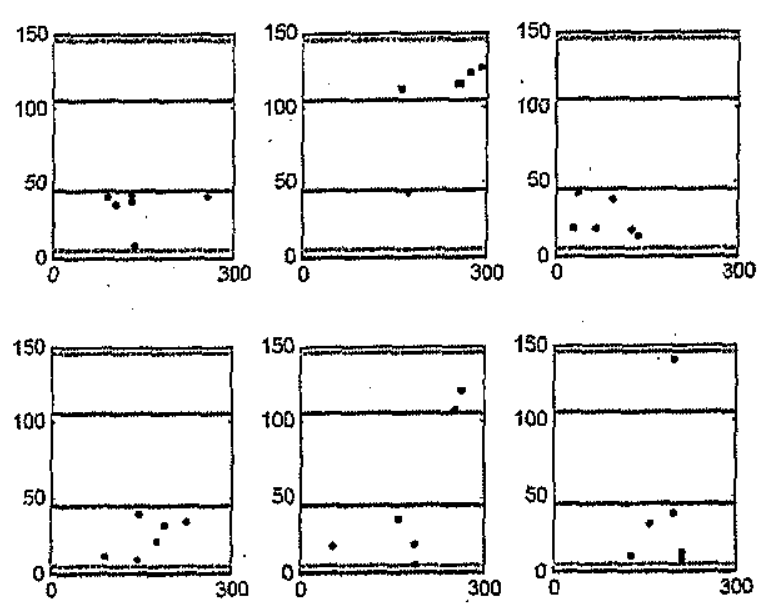

Fig. 9. Locations of different facilities for nine different solutions
Finding a good distribution of solutions near the Pareto optimal front in small computational time is a dream of multi-objective $\mathrm{EA}$ researchers and practitioners. In this paper a new optimization system is presented, which is based on the concept of coevolution and modified genetic operator that maintain the feasibility. Also it is based on the iterative external archive of non-dominated solutions which gets iteratively updated. The methods keep track of all the feasible solutions found during the optimization and therefore do not have any restrictions on the number of Pareto-optimal solutions found.

In this example, six Pareto points were obtained. These six Pareto points constitute a problem to decision maker (DM) conceming the point that must be chosen. However, this is the basic characteristic feature of TOPSIS method. The only needed is the weights that reflect the importance of each objective from the view of the DM.

For example, if the weights are $\left\{\sum_{i=1}^{k=2} w_{i}=1 \mid w_{1}=0, w_{2}=1\right\}$ then the solution numbered 6 is considered, as in Fig. 7, which means that the DM is interesting in the second objective only. Also, for $\left\{\sum_{i=1}^{k=2} w_{i}=1 \mid w_{1}=1, w_{2}=0\right\}$ the solution numbered 1 is chosen, which means that the $\mathrm{DM}$ is interesting in the first objective without consideration of the second objective. However, for $\left\{\sum_{i=1}^{k=2} w_{i}=1 \mid w_{1}=0.3, w_{2}=0.7\right\} \quad$ the solution numbered 4 is preferred. It is concluded that TOPSIS techniques are used to help the DM to extract the best compromise solution from a finite set of alternatives. Also it guarantees an optimal distribution of solutions.

\section{CONCLUSIONS}

In this paper, an optimization system to solve the Multi-objective construction site layout (MCSL) problem was proposed. MCSL is considered with assigning of predetermined facilities into a number of predetermined places. The problem has two objectives to minimize overall distance and to reduce the number of intersecting paths between different facilities. The proposed approach has two characteristic features. Firstly, a new chromosome's structure, which is adopted as it is capable to representing all possible feasible solutions, is introduced. Secondly, the algorithim is an iterative multi-objective genetic algorithm with an external population of Pareto optimal solutions that best conform a Pareto front. Moreover to help the decision makers to extract the best compromise solution from a finite set of alternatives a TOPSIS 
method is implemented. A simple case study for a highway project is utilized to demonstrate the application of the model. The main features of the proposed algorithm could be summarized in the following points:

- The proposed approach has been effectively applied to solve the MCSL, with no limitation in handing higher dimensional problems.

- The non-dominated solutions in the obtained Pareto-optimal set are well distributed and have satisfactory diversity characteristics.

- Implementation of modified genetic algorithm operator (Crossover-Mutation) maintains the chromosome feasibility.

- TOPSIS techniques are used to help the decision maker to extract the best compromise solution from a finite set of alternatives.

- Simulation results verified the validity and the advantages of the proposed approach.

- The results suggest that the proposed optimization system is better applicable for solving real-world application problems.

\section{REFERENCES}

[1] Tam, C.M. and Tong, T.K.L. (2003). "GA-ANN model for optimizing the locations of tower crane and supply points for high-rise public housing Construction." Journal of Construction Management and Economics, Vol. 21(3), pp. 257266.

[2] Osman, H.M., Georgy, M.E. and Ibrahim, M.E. (2003). "A hybrid CAD-based construction site layout planning system using genetic algorithms." Automation in Construction, Vol. 12(6), pp. 749764.

[3] Mawdesley, M.J. and Al-Jibouri, S.H. (2003). "Proposed genetic algorithms for construction site layout." Engineering Applications of Artificial Intelligence, vol. 16 (5-6), pp. 501-509.

[4] Cheung, S., Tong, T.K. and Tam, C. (2002). "Site pre-cast yard layout arrangement through genetic algorithms." Automation in Construction, Vol. II(1), pp 35-46.

[5] Elbeltagi, E. and Hegazy, T. (2001). "A hybrid AIbased system for site layout planning in construction." Journal of Computer-Aided Civil and Infrastructure Engineering, Vol. 16(2), pp. 7993.

[6] Tommelein, I.D. (1989). "SightPlan-an expert system that models and augments human decisionmaking for designing construction site layout." $\mathrm{PhD}$ dissertation, Department of Civil Engineering, Stanford University, Stanford, Califomia, USA.

[7] Tommelein, I.D., Levitt, R.E. and Hayes-Roth, B. (1992). "SightPlan model for site layout." Journal of Construction Engineering, ASCE, Vol. 118(4), pp 749-766.
[8] Yeh, I-C. (1995). "Construction site layout using annealed neural networks." Journal of Computing in Civil Engineering, ASCE, Vol. 9(3), pp. 201208.

[9] Zouein, P.P. and Tommelein, I.D. (1.999). "Dynamic layout planning using a hybrid incremental solution method." Journal of Construction. Engineering and Management, ASCE, Vol. 125(4), pp. 400-408.

[10] Cheng, M. and O'Connor, J. (1996). "ArcSite: enhanced GIS for construction site layout." Journal of Construction Engineering and Management, ASCE, Vol. 122(4), pp. 329-336.

[11] Hegazy, T. and Elbeltagi, E. (1999). "EvoSite: An evolution-based model for site layout planning." Journal of Computing in Civil Enginnering, ASCE, Vol. 13(3), pp. 198-206.

[12] AbdelRazig, Y., El-Gafy, M. and Ghanem, A. (2006). "Dynamic construction site layout using ant colony optimization." $85^{\text {th }}$ Annual Meeting of Transportation Research Board, Washington, D.C., USA.

[13] El-Rayes, K. (2009). "Dynamic site layout planning using approximate dynamic programming." Journal of Computing in Civil Enginnering, ASCE, Vol. 23(2), pp. 1110-127.

[14] Zouein, P.P., Harmanani, H. and Hajar, A. 2002. "Genetic algorithm for solving site layout problem with unequal-size and constrained facilities," Journal of Computing in Civil Engineering, Vol. 16(2), pp. 143-151.

[15] Hwang, C.L. and Yoon, K., (1981). "Multiple Attribute Decision Making: Methods and Applications." Springer-Verlag, Berlin.

[16] Miettinen K., (2002). "Non-linear multi-objective optimization." Dordrecht: Kluwer Academic Publisher.

[17] Osman, M.S., Abo-Sinna, M.A., and Mousa, A.A. (2006). "IT-CEMOP: An Iterative co-evolutionary algorithm for multi-objective optimization problem with nonlinear constraints." Journal of Applied Mathematics \& Computation (AMC), Vol. 183, pp. 373-389.

[18] Holland, J.H. (1975). "Adaptation in Natural and Artificial Systems." The University of Michigan Press. Ann Arbor, Michigan,

[19] Goldberg, G.E., (1991). "Genetic Algorithms in Search, Optimization and Machine Learning." Addison Wesley Publishing Company.

[20] Michalewicz, Z., (1996). "Genetic Algorithms + Data Structures = Evolution Programs." SpringerVerlag, $3^{\text {rd }}$ Edition.

[21] Deb, K., (1991). "Multi-objective optimization using evolutionary algorithms". NY, USA: Wiley.

[22] Olson D.L., (2004). "Comparison of Weights in TOPSIS Models, Mathematical and computer Modeling." Vol. 40, pp.721-727. 